\title{
Vestigios del recurso costero en el sitio arqueológico de Teopancazco, Teotihuacan, Estado de México
}

\author{
Bernardo RodRíguez GALICIA \\ Instituto de Investigaciones Antropológicas, Universidad Nacional Autónoma de México \\ sanber65@hotmail.com \\ Raúl VALAdEz AzÚA \\ Instituto de Investigaciones Antropológicas, Universidad Nacional Autónoma de México \\ raul_valadez@hotmail.com
}

Recibido: 13 de octubre de 2011

Aceptado: 31 de febrero de 2013

\begin{abstract}
RESUMEN
El presente artículo es una síntesis del trabajo de investigación Captura, preparación y uso diferencial de la ictiofauna encontrada en el sitio arqueológico de Teopancazco, Teotihuacan, el cual hace hincapié en el hallazgo de diferentes huesos de peces encontrados en el contexto arqueológico de Teotihuacan, algo realmente inusual en esta área del altiplano central mexicano. El sitio arqueológico donde se efectuó el hallazgo de dichos materiales es el centro del barrio de Teopancazco, lugar que se ubica en la periferia del centro ceremonial, y turístico, de la mítica «Ciudad de los dioses». Aquí la presencia de variedades ícticas como el pez bobo (Joturus pichardi), el huachinango (Lutjanus $\mathrm{sp}$ ), robalo (Centropomus $\mathrm{sp}$ ), mojarras (Diapterus sp) o barracudas (Sphyraena barracuda), por citar algunas, rompe con la falsa idea de que en época prehispánica los antiguos teotihuacanos no tenían acceso, ni gusto, por los recursos costeros.
\end{abstract}

Palabras clave: Centro de barrio, peces, recursos costeros, golfo de México.

\section{Vestiges of the Coastal Resource in the Archaeological Site of Teopancazco, Teotihuacan, State of Mexico}

\begin{abstract}
This research is a synthesis of the work Capture, preparation, and differential use of the ichthyofauna found in the archaeological site of Teopancazco, Teotihuacan, which emphasizes the discovery of different bone fish found in the archaeological context of Teotihuacán, something truly unusual in this area of the central Mexican plateau. The archaeological site where took place the finding of these materials is the center of neighborhood of Teopancazco, site that is located on the periphery of the ceremonial center, and tourism, the mythical «City of the gods». Here the presence of fish varieties such as bobo mullet (Joturus pichardi), red snapper (Lutjanus sp), common snook (Centropomus sp), mojarra (Diapterus sp) or barracuda (Sphyraena barracuda), to mention some, breaks with the false idea that, at pre-Hispanic era, the ancient Teotihuacans had no access to, or taste for, coastal resources.
\end{abstract}

Key words: Center of neighborhood, fish, coastal resources, Gulf of Mexico

Sumario: 1. Introducción. 2. Ubicación del sitio de estudio. 3. Objetivos y método de la investigación. 4. Resultados de los análisis. 5. Disposición de los recursos costeros en la Ciudad de los Dioses. 6. Procedencia de los recursos costeros. 7. Captura y preparado de los peces. 8. Ecosistemas de procedencia de la fauna costera. 9. Cosmovisión costera en la Ciudad de los Dioses. 10. Consideraciones finales. 11. Referencias bibliográficas.

\section{Introducción}

La arqueozoología, como rama de la investigación arqueológica y zoológica, tiene como objetivo abordar los aspectos socioeconómicos y culturales a través del estudio 
contextual de la fauna hallada en un yacimiento arqueológico, así como las características paleoecológicas que le rodean. El análisis de los materiales fáunicos tiene el propósito de proporcionar al arqueólogo información sobre la anatomía, taxonomía, geografía de distribución, ecología, biología y explotación diferencial del recurso animal que se ha recuperado en el sitio de excavación, dándole las posibles alternativas de su uso en por lo menos cuatro rubros:

1. Consumo: principalmente de las especies animales que tienen un uso alimentario o utilitario ${ }^{1}$.

2. Ornamento: especies animales que sirvieron para ser explotadas como fuente abastecedora de plumas, piel, hueso, concha y otros elementos anatómicos, para adornar tocados, trajes, estandartes y vestimentas ceremoniales.

3. Cautiverio: sobre todo de organismos que pudieron haber vivido como mascotas, o animales que sirvieron como fuente de abastecimiento de carne o materia prima para obtener de ellos algún elemento anatómico para servir de amuleto, adorno o herramienta de trabajo.

4. Comercio: sobre todo en el tráfico de animales con otras ciudades que, por su ubicación ecológica y geográfica, requerían de ser transportados como fauna «exótica» que sirviera como elemento de intercambio, pago, tributo, ofenda o requerimiento ceremonial que hacían los pueblos aliados, subyugados o conquistados.

Para llevar a cabo estos estudios es necesario entender la importancia de cada dato potencial y enfocarlo desde múltiples puntos de vista, sobre todo en aquellos en los que los criterios antropológicos y biológicos manifiesten una tendencia afín al valor cultural de los usos y costumbres de los pueblos, especialmente en la etnología de las comunidades actuales.

Uno de los sitios que más interés ha despertado al respecto de lo anterior es la antigua ciudad de Teotihuacan, Estado de México. En ella se han podido datar, a lo largo de 1150 años, al menos seis etapas de ocupación humana (Cuadro 1). Esta mega-urbe prehispánica ha sido objeto de investigación arqueológica desde hace prácticamente un siglo, aunque sólo en las últimas décadas se han abierto las posibilidades de que el estudio de los espacios arqueológicos involucre el conocimiento d las relaciones entre el hombre y la fauna circundante (Manzanilla 1997; Padró 2002; Rodríguez 2006).

El estudio de la relación hombre-animal en el pasado corresponde a la arqueozoología; esta línea de investigación que deambula entre lo cultural y lo biológico ha permitido establecer qué animales fueron aprovechados como fuente de alimento, abastecedores de materia prima para actividades rituales-ceremoniales $u$ ofrendas, elementos tributarios, unidades decorativas, utensilios de trabajo, fuente de obtención de materia prima, hueso, pelo o pluma, para adorno de vestimentas, tocados, estandartes, etc.

En Teotihuacan, lo anterior no es la excepción y, en su condición de urbe cosmopolita, es esperable una serie de pautas de uso diferencial de los animales, incluyendo un conocimiento y aprovechamiento de los recursos fáunicos, y un conocimiento de

${ }^{1}$ Utilitario después de haber servido como alimento o como animales disecados empleados en la medicina tradicional, como se puede ver hoy en día en los mercados y tianguis de México, o como materia prima en la confección y elaboración de vestimentas. 
Cuadro 1: Fases de la cronología teotihuacana, indicando con negrita el período de mayor ocupación para Teopancazco (Manzanilla 2000; Millon 1973).

\begin{tabular}{ll}
\hline \multicolumn{2}{c}{ Cronología de Teotihuacan } \\
\hline Período Formativo Tardío y Terminal & 500 a.C. -0 \\
Fase Tzacualli & $0-100$ d.C. \\
Fase Miccaotli & 100 d.C. -200 d.C. \\
Fase Tlamimilolpa & $\mathbf{2 0 0}$ d.C. $-\mathbf{3 5 0}$ d.C. \\
Fase Xolalpan & $\mathbf{3 5 0}$ d.C. $-\mathbf{5 5 0}$ d.C. \\
Fase Metepec & $\mathbf{5 5 0}$ d.C. $-\mathbf{6 5 0}$ d.C. \\
Período Epiclásico & 650 d.C. -850 d.C. \\
Período Posclásico & 850 d.C. -1500 d.C. \\
\hline
\end{tabular}

los ambientes de donde provenían. Respecto al primero, los huesos son una clara evidencia de la dinámica del uso de los animales, desde su crianza hasta el empleo de diversas unidades anatómicas para la elaboración de objetos utilitarios (Manzanilla et al. 2009; Valadez 2011). Respecto al conocimiento de los ambientes, desde las primeras décadas del siglo XX se determinó la existencia de materiales faunísticos procedentes de regiones fuera de la Cuenca de México, básicamente conchas marinas, o bien iconografía vinculada con especies que no habitan la región, como sería el caso del jaguar o el quetzal (Valadez 1992a). No obstante, no fue hasta el final de ese siglo que aparecieron los primeros restos de vertebrados alóctonos (Valadez 1992a, 1992b, 1993) y, por raro que parezca, la reacción inicial de los arqueozoologos mexicanos fue rechazar la posibilidad de que alguna vez los teotihuacanos hubieran tenido la habilidad de impulsar el flujo de animales desde regiones distantes, como serían las costas mesoamericanas, hacia el altiplano central.

Los primeros hallazgos de vertebrados foráneos consistieron principalmente en partes aisladas que denotaban un valor simbólico por sí mismas (Valadez 1992b), lo cual dejaba clara la opción de que parte de este flujo de animales eran sencillamente objetos manufacturados con una importancia intrínseca. Posteriormente se descubrieron restos de animales completos que no dejaban lugar a dudas acerca de que, dentro de la ciudad de Teotihuacan, habían circulado especies tan impresionantes como los jaguares.

Sin duda el espacio teotihuacano en el cual se ha detectado y estudiado la mayor concentración de organismos alóctonos es el centro de barrio de Teopancazco (Manzanilla et al. 2009). La colección incluye, en su mayoría, peces de origen costero, que han aportado datos sobre los métodos de pesca, preparación y uso diferencial de este recurso natural; del mismo modo también se ha podido identificar un fragmento de espina de erizo de mar (Eucidaris thouarsii), pinzas de dos especies de cangrejos (Cardisoma ca. guanhumi y Gecarcinus ca. lateralis) y placas sub-dérmicas de cocodrilo (Crocodylus sp).

La alta concentración de estos elementos anatómicos, pero sobre todo de los huesos de peces, implica dar un enorme salto en el conocimiento de lo que era el manejo de los recursos animales provenientes de regiones fuera de la Cuenca de México, pues permite pasar de la captura de una cría de jaguar, en la Sierra Madre Oriental, a la captura de peces, cocodrilos o cangrejos de la costa, rompiendo con la falsa idea de que en Teotihuacan no se tenía un aprovechamiento habitual de los recursos costeros, 
e impulsando la idea de que la cultura teotihuacana tenía un acceso y conocimiento, casi ilimitado, de los bienes naturales mesoamericanos:

«La fauna costera encontrada en Teotihuacan, particularmente en Teopancazco, tiene un gran valor cultural, pues su presencia implica una estrecha relación del hombre con el ambiente acuático; pudiendo decir que la interacción, hombre-peces, inicia con la observación de los organismos, su captura, preparación y hasta su traslado a otros lugares fuera de su ambiente natural» (Rodríguez 2010: 3).

\section{Ubicación del sitio de estudio}

La Cuenca de México se localiza entre los $19^{\circ} 02^{\prime}$ y $20^{\circ} 12^{\prime}$ latitud norte y $98^{\circ} 28^{\prime}$ y $99^{\circ} 32^{\prime}$ de longitud oeste, estableciendo el límite sur de la Altiplanicie Mexicana (Valadez 1983: 7). Su longitud mayor va desde el Volcán Ajusco hasta la Sierra de Pachuca, aproximadamente a $130 \mathrm{~km}$; su anchura mayor se acerca a los $90 \mathrm{~km}$; la superficie total supera los $9500 \mathrm{~km}^{2}$ y su zona más baja se encuentra $2235 \mathrm{msnm}$ (Rzedowski y Calderón 1979; Valadez 1983: 7).

El lugar de donde provienen los restos de fauna costera está en la antigua Teotihuacan, Estado de México, ciudad prehispánica que se localiza a unos $50 \mathrm{~km}$ de distancia al noreste de la Ciudad de México (Rodríguez 2010: 74), siendo Teopancazco el sitio arqueológico específico de donde se extrajeron los restos arqueozoológicos. Técnicamente, Teopancazco se localiza al sureste de la Ciudadela (Figura 1), ocupando el Cuadro S2 E2 del mapa de Millon (1973: 70) y posiblemente sus dimensiones hayan alcanzado unos 60 × 60 m; se constituyó, según este autor, en la fase Tlamimilolpa (200-350 d.C.) y su ocupación continúa hasta la Metepec (550-650 d.C.) (ver Cuadro 1).

Este Centro de Barrio cuenta con un Patio Central de aproximadamente $14 \mathrm{~m}$ de lado, así como con algunos cuartos con pintura mural donde se observan procesiones de sacerdotes frente a santuarios y algunos guerreros (Gamio 1922, primera parte: 156-157; Lombardo 1996: 43, 53; Manzanilla 2006, 2007; Rodríguez 2010: 75).

\section{Objetivos y método de la investigación}

Actualmente existe un gran vacío en la investigación del recurso costero importado en poblaciones humanas extintas del Altiplano Central; Teotihuacan no es la excepción y ante la evidencia suscitada con el hallazgo de tan alta concentración de elementos anatómicos, que corresponden a peces o cangrejos marinos, la investigación plantea la posibilidad de que tal concentración sea el reflejo de organismos que provenían de la costa más cercana al sitio de estudio, es decir, del Golfo de México.

Así, algunos de los huesos, sobre todo aquellos que indican presencia de individuos completos, dejan ver que estos animales tuvieron una incuestionable importancia en actividades rituales-ceremoniales y de consumo humano como alimento; también se puede inferir que para el traslado de algunos de los organismos se establecieron redes de vinculación «comercial» entre estas distantes regiones, con el propósito de transportar productos costeros hasta su destino final, pudiendo inferir que quizás los antiguos pobladores de las costas del Golfo de México recurrieran a 


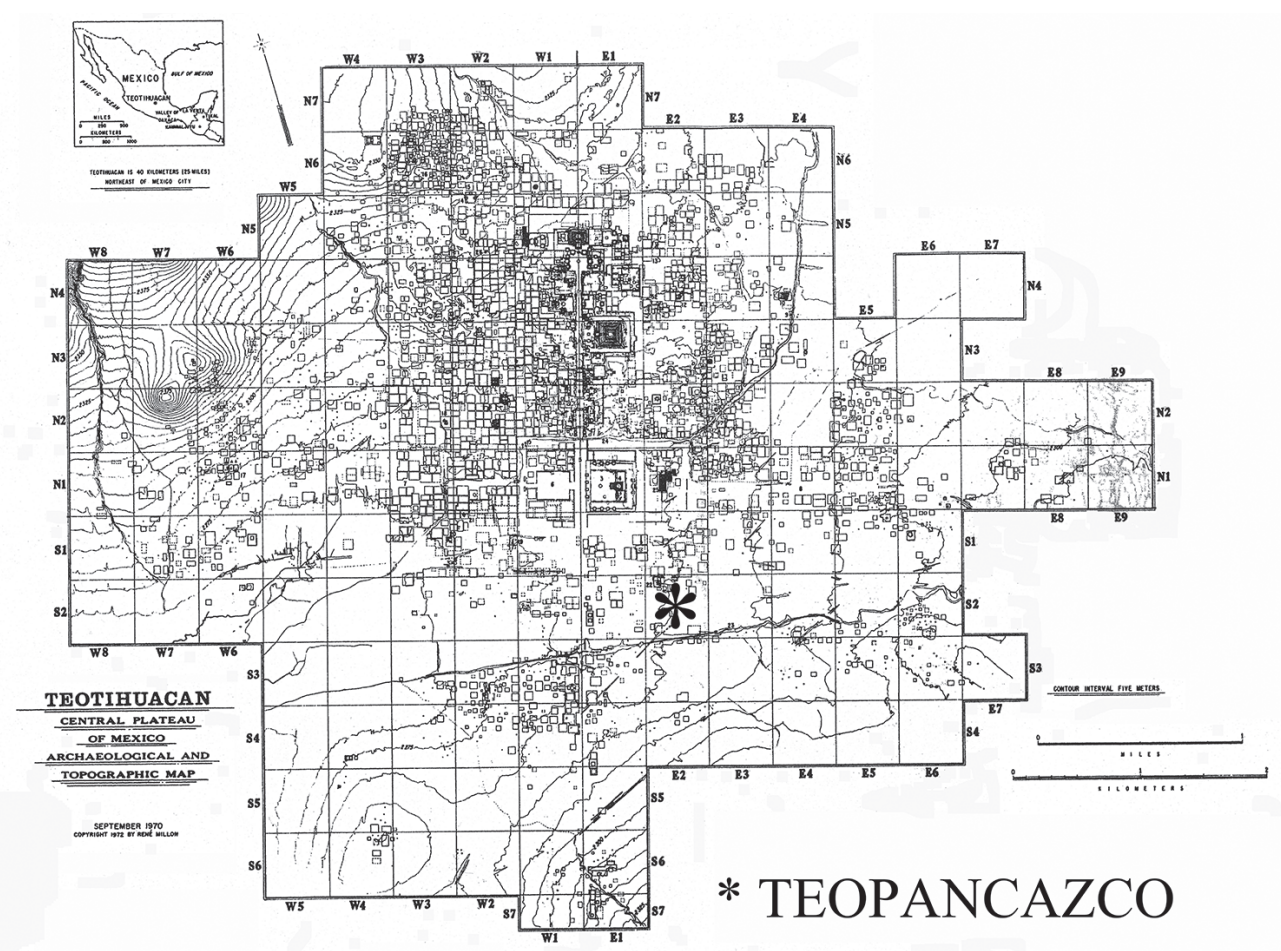

Figura 1: Ubicación de Teopancazco (S2 E2; tomado y modificado del Mapa de Millon 1973).

técnicas de preservación (salado, ahumado o guisado), en algunas de las variedades de peces transportadas a Teotihuacan. Con base en lo anterior los objetivos de la presente investigación son:

1. Exponer y sustentar el aprovechamiento de los recursos costeros empleados por la sociedad teotihuacana a partir del análisis de los restos animales, las fuentes etnohistóricas y el ciclo de vida de los organismos.

2. Determinar los ecosistemas de procedencia de los organismos identificados, así como inferir sobre los métodos de captura de los mismos.

3. Evaluar la importancia del recurso costero en la sociedad teotihuacana a través del análisis y correlación de los ciclos de vida de los animales identificados.

Los materiales arqueozoológicos que se trabajaron en este estudio corresponden a las temporadas de excavación efectuadas entre los años 1997 a 2005 del proyecto Teotihuacan: elite y gobierno (Teopancazco), dirigido y coordinado por la Dra. Linda Manzanilla. Estos materiales óseos fueron trasladados para su análisis, al Laboratorio de Paleozoología del Instituto de Investigaciones Antropológicas de la Universidad Nacional Autónoma de México.

En general el trabajo metodológico da inicio con la limpieza, restauración -si así lo requiere el hueso-, etiquetado y observación de los elementos óseos al microscopio, (Estereoscopio ZEIZZ-KL 200), con el propósito de detectar posibles alteraciones como huellas de corte, pulido, tallado, masticado, sometimiento al calor indirecto 


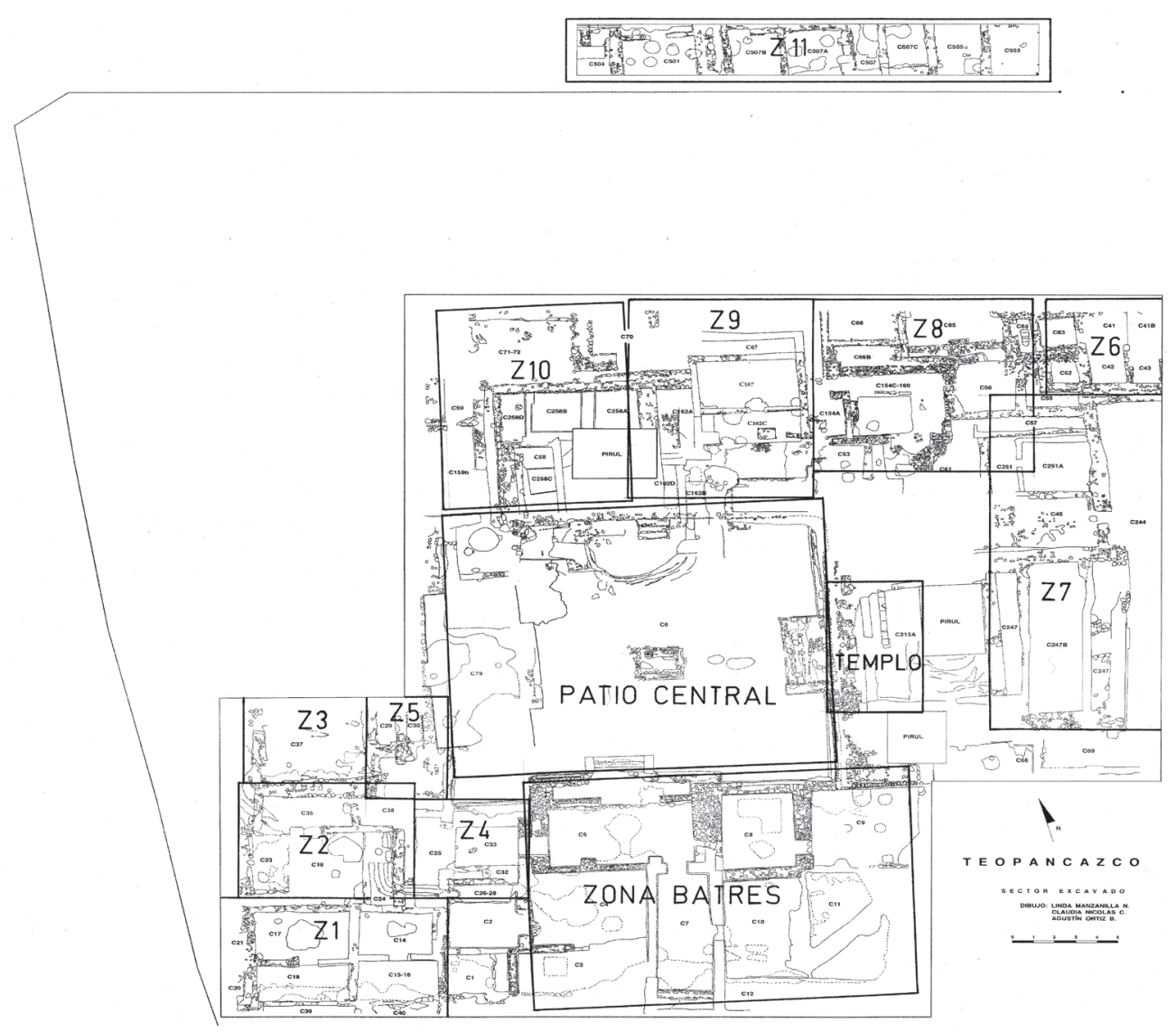

Figura 2: Imagen en planta de Teopancazco, dividido por sectores.

(cuando el animal aun tiene «paquetes musculares») o directo (si el hueso es sometido o expuesto, conciente o inconscientemente, a alguna fuente de calor externa), etc.

El trabajo de identificación anatómica y positiva-taxonómica de los materiales fáunicos continúa con los siguientes principios básicos:

1. Consulta de bibliografía especializada en anatomía, osteología, y de distribución geográfica, ecológica y etnobiológica, de las especies «posibles» a identificar.

2. Consulta de colecciones científicas de referencia, o en su defecto de la preparación de ejemplares que sirvan para apoyar la identificación positiva.

Como parte de la metodología, es indispensable la obtención del índice Número Mínimo de Individuos (NMI), ya que de él depende conocer cuál fue la abundancia de cada una de las especies identificadas (O'Connor 2000); para lograrlo se efectuaron además las siguientes acciones:

1. El establecimiento del número o lateralización de los huesos pares e impares.

2. Análisis de la distribución espacial, general, de los restos animales en el sitio de estudio. 
3. Establecimiento de una correlación anatómica, con base en la textura, tamaño, color y, en algunos casos, alteración efectuada por la acción del hombre, considerando huesos cercanos en los cuadrantes de la retícula de excavación.

4. Finalmente se llevó a cabo una correlación del contexto arqueológico en cuanto a los materiales fáunicos identificados: ubicación de rellenos, cuartos, áreas de actividad, apisonados, pisos, fosas, entierros, etc.

Respecto a la determinación de la talla de los peces, ésta se obtuvo a partir de la comparación directa de los huesos arqueoictiológicos y los elementos óseos correspondientes a organismos actuales; es decir, se emplearon esqueletos de peces de comparación que fueron preparados previamente en el laboratorio de Paleozoología, no sin antes obtener de ellos las medidas morfológicas comunes para todos los peces, y las propias de la especie, peso, muestras de escamas, y otolitos (Polaco y Guzmán 1997: 53).

Es importante indicar que, para poder emprender el análisis de la fauna costera sobre todo de los peces identificados, se procedió a la sectorización del sitio de estudio, con base en lo propuesto por Pecci (2000) y Rodríguez (2006: 52-53); lo anterior con el propósito de elaborar mapas de ubicación de los materiales dentro de Teopancazco (Figura 2) y conocer la abundancia y variedades de organismos detectados en espacios específicos ${ }^{2}$.

\section{Resultados de los análisis}

Con respecto al material no íctico, los resultados del análisis permitieron contabilizar una espina de un erizo de mar (Eucidaris thouarsii) la cual presenta evidencia de haber sido sometida a trabajo de tallado. Este organismo, a decir del Dr. Solís (2005), es de distribución exclusiva del Pacifico oriental, desde California hasta el Ecuador, incluyendo Isla del Coco y Galápagos. Con respecto a los cangrejos, identificados por Román y Martínez (2005), se contabilizaron nueve fragmentos de pinzas, de por lo menos cinco individuos, de cangrejos rojos (Gecarcinus ca. lateralis) y una de cangrejo azul (Cardisoma ca. guanhumi), ambos decapados oriundos de las costas del Golfo de México.

En lo que corresponde a los vertebrados identificados se tienen treinta placas subdérmicas de cocodrilo (Crocodylus sp) y 1098 huesos correspondientes a cráneo, esqueleto apendicular y columna vertebral de diferentes variedades de peces (Cuadro 2). Al respecto del primero, la Mtra. Alicia Blanco (comunicación personal) menciona:

«Por la forma y tamaño de las placas, éstas tienen más probabilidad de ser de Crocodylus moreletii, que es de talla pequeña en comparación con Crocodylus acutus que

2 Es importante mencionar que se estuvo en comunicación constante con el Mtro. en Ciencias Edmundo Teniente, de la Escuela Nacional de Ciencias Biológicas del IPN, y con la Dra. Ana Fabiola Guzmán, de la Subdirección de Laboratorios y Apoyo Académico del INAH, para la corroboración y asesoría en la identificación taxonómica de las especies pesqueras; mientras que la identificación de la espina de erizo de mar fue efectuada por el Dr. Francisco Solís y los cangrejos por el Dr. Ramiro Román y el Mtro. en Ciencias Mario Martínez del Instituto de Ciencias del Mar y Limnología de la UNAM. 
es por mucho más grande; esta primera especie es propia del Golfo de México y su distribución abarca desde el estado de Tamaulipas, por la costa del Golfo de México, y llega hasta la península de Yucatán» (Rodríguez 2010: 187-188).

En cuanto a los materiales ícticos el análisis permitió establecer una considerable cantidad de vértebras, espinas, huesos craneales y fragmentos óseos que pertenecen a por lo menos 17 peces que no pudieron ser identificados, 13 organismos reconocidos en el rango taxonómico de familia, 26 en género y 43 individuos que pudieron identificarse a nivel de especie; sumando un Número Mínimo de Individuos (NMI) igual a 99. Al respecto de lo anterior, es importante señalar que, siguiendo los lineamientos para el establecimiento del NMI sugeridas por Bökönyi (1970), quien le da mayor peso a la lateralización de los huesos pares, el presente estudio aplicó parámetros de dispersión ósea de elementos anatómicos en el sitio de estudio y los parámetros culturales y naturales que permitieran conformar individuos, aunque éstos quedaran identificados a nivel de familia o como peces no identificados.

Cuadro 2: Lista de vertebrados alóctonos y de invertebrados no moluscos, identificados a taxa género y especie.

\begin{tabular}{|c|c|c|c|}
\hline Géneros y especie & MNI & $\begin{array}{c}\text { Algunos de los restos biológicos } \\
\text { descubiertos }\end{array}$ & Origen \\
\hline Eucidaris thouarsii & 1 & Espina tallada & Costa del Pacifico mexicano \\
\hline Cardisoma guanhumi & 1 & Pinza & Costa del Golfo de México \\
\hline Gecarcinus lateralis & 6 & Nueve pinzas & Costa del Golfo de México \\
\hline Crocodylus moreletii & 1 & 30 placas sub-dérmicas & Costa del Golfo de México \\
\hline Lutjanus sp & 13 & Huesos diversos & Ambos litorales mexicanos \\
\hline Lile sp & 2 & Esqueletos completos & Costa del Pacifico mexicano \\
\hline Diapterus sp & 1 & Espina anal y radio & Ambos litorales mexicanos \\
\hline Ictalarus sp & 4 & $\begin{array}{l}\text { Espina dorsal, vértebras, subopercu- } \\
\text { lar y cleitros }\end{array}$ & Ambos litorales mexicanos \\
\hline Eucinostomus sp & 1 & Espinas costal, anal y dorsal. & Ambos litorales mexicanos \\
\hline Centropomus sp & 2 & $\begin{array}{l}\text { Cuerpos vertebrales, cuadrado iz- } \\
\text { quierdo, opérculo derecho, vértebra } \\
\text { caudal y diversas espinas }\end{array}$ & Ambos litorales mexicanos \\
\hline Caranx sp & 2 & $\begin{array}{l}\text { Hiomandibular derecho, espinas dor- } \\
\text { sales, opérculo y vértebras }\end{array}$ & Ambos litorales mexicanos \\
\hline Carcharinus leucas & 1 & Diente superior & Costas del Golfo de México \\
\hline Joturus pichardi & 36 & Esqueletos completos & Costas del Golfo de México \\
\hline Epinephelus nigritus & 1 & $\begin{array}{l}\text { Vértebra, postemporal derecho, espi- } \\
\text { na dorsal y dentario derecho }\end{array}$ & Costas del Golfo de México \\
\hline Mycteroperca bonaci & 1 & Cleitro izquierdo & Costas del Golfo de México \\
\hline Caranx hippos & 2 & $\begin{array}{l}\text { Hiomandibular, espina dorsal, opér- } \\
\text { culo y vértebra caudal }\end{array}$ & Ambos litorales mexicanos \\
\hline Bairdiella ronchus & 1 & $\begin{array}{l}\text { Vértebra caudal, espina anal y espina } \\
\text { hemal }\end{array}$ & Costas del Golfo de México \\
\hline Sphyraena barracuda & 2 & Dos dentarios derechos & Costas del Golfo de México \\
\hline
\end{tabular}


A partir de ello, las zonas o sectores ${ }^{3}$ que contabilizan un mayor NMI ícticos son: zona 7 con 27 individuos, zona 9 con 23, zona 10 con 14, zona 8 y el Patio Central con 9 cada uno, el Templo con 5, zona 2 con 4 , zona 11 con 3 y el resto de los sectores 1, 3, 4, 6 y Batres con tan sólo un individuo en cada caso. Así también se observa, sin tomar en cuenta a los peces no identificados, una mayor presencia de peces bobo (Joturus pichardi) y huachinangos (Lutjanus sp); el resto de peces tiene una representación que va de los cuatro hasta un solo individuo.

Respecto de los huesos identificados anatómicamente, estos corresponden a 41 tipos, un diente, otolitos, escamas y un fragmento de piel, inmerso en una matriz de suelo como «polvo blanco o talco». Así también se descubrieron conjuntos de huesos que correspondieron a ejemplares completos $(85-86 \%$ del esqueleto) de sardina (Lile sp.). En general la frecuencia ósea parte del total de huesos contabilizados, 1098 elementos (sin considerar los huesos de la sardina ${ }^{4}$ ). Del total de huesos, los que mayor presencia tienen son las vértebras con $395(35,97 \%)$; las costillas con $130(11,83 \%)$; los radios o rayos con $75(6,83 \%)$; los fragmentos de cráneo con $51(4,64 \%)$; los operculares con 50 (4,55\%); le siguen las espinas dorsales con $49(4,46 \%)$, para posteriormente continuar con lo que se identificó como hueso plano con $38(3,46 \%)$; los cleitrum son $37(3,37 \%)$, los preoperculares $35(3,18 \%)$, del premaxilar son 30 $(2,73 \%)$, articulares $29(2,64 \%)$, el hiomandibular con $23(2,09 \%)$, los dentarios son $15(1,36 \%)$, los paraesfenoides, al igual que las espinas laterales son $14(1,27 \%)$, cada uno, los cuadrados $13(1,18 \%)$, el postemporal $12(1,09 \%)$, el hueso urohial está en 11 ocasiones $(1,01 \%)$. En contraparte hay 24 tipos de huesos que no sobrepasan el $1 \%$, entre ellos dientes, el prevomer, el basipterigium, el coracoides, el postcleitrum, el ceratobranquial, el faringeobranquieles y el supraoccipital.

En lo que se refiere a la frecuencia ósea, el sector que tiene la mayor concentración es la zona 9 con 495 huesos (45,08\%), le sigue el sector 10 con 341 (31,05\%), mientras que en tercer lugar lo ocupa la zona 7 con 103 (9,38\%), le continúa la zona 8 con 79 (7,19\%), posteriormente el Patio Central con 27 (2,45\%), el Templo con 21 $(1,91 \%)$, la zona 2 con $16(1,45 \%)$, los sectores 4 y 11 con $4(0,36 \%)$, cada una, la zona Batres con $3(0,27 \%)$, la 3 y 6 con $2(0,18 \%)$ y el de menor presencia es el sector 1 con un único elemento $(0,09 \%)$.

Así también resulta importante mencionar que durante el análisis de los huesos se observó una frecuente alteración, concentración en forma de «costras» en la superficie de diferentes elementos anatómicos o, como en el caso de la piel inmersa en el «polvo blanco» o «talco». Con base en lo anterior se sugirió efectuar, a petición del Dr. José Luís Ruvalcaba, del Instituto de Física de la UNAM, en una muestra de 10 elementos, un análisis químico por difracción de Rayos X (RX) y Microscopía Electrónica de Barrido (MEB), con el propósito de conocer qué eran esas concentraciones de «polvo blanco» y «costras» en los huesos. Los resultados permitieron establecer la existencia de diferentes tipos de sales, derivadas del sodio $(\mathrm{Na})$, potasio $(\mathrm{K})$, o

\footnotetext{
3 Es importante señalar que en el presente estudio se hace un uso indistinto del término zona o sector, pues se refieren a lo mismo.

4 La razón de no incluir los huesos de sardina tiene como fundamento el no incrementar considerablemente la frecuencia ósea general del total de individuos contabilizados.
} 
magnesio $(\mathrm{Mg})$, que pudieron emplearse de manera «indirecta ${ }^{5} »$ en la preparación, salado, ahumado o secado al sol de los peces, antes de su traslado al Altiplano Central, evidenciando concentraciones de sales poco usuales en los huesos, sobre todo en aquellos donde se observaban cambios en la coloración natural del elemento óseo.

\section{Disposición de los recursos costeros en la Ciudad de los Dioses}

Los peces tienen diferentes planos anatómicos, su cuerpo generalmente se divide en cabeza, tronco y cola; pese a lo anterior resulta difícil poder decir, externamente, dónde empieza uno y dónde termina el otro, anatómicamente los peces, al igual que el resto de los vertebrados, son regionalizados de acuerdo a su forma, función, posición o número de apéndices, siendo importante la ubicación de las regiones de acuerdo a los planos anatómicos, ya que ello ayudó a plantear posibles relaciones entre zonas del cuerpo, determinación de NMI y uso de los peces en Teopancazco.

Los resultados mencionados anteriormente y la regionalización anatómica del cuerpo de los peces -región craneal, esqueleto apendicular y columna vertebral-, permiten establecer que la segmentación anatómica que aparece con mayor frecuencia es la columna vertebral con un total de 533 elementos óseos, poco más del 52\%; le sigue la región del cráneo con 270 huesos y un valor cercano al $27 \%$; y, por último, el esqueleto apendicular con un total de 206 huesos y una frecuencia del $20 \%$.

Se puede concluir, por tanto, que la región anatómica que tiene una mayor presencia es la columna vertebral, según los planos sagitales; sin embargo también se presenta una nada despreciable cantidad de huesos que comprenden la región de la cabeza de estos vertebrados. Los datos indican que existe una sincronía entre las regiones del cuerpo de los peces encontrados en Teopancazco y las frecuencias de elementos óseos que se observan en un esqueleto completo, pudiendo establecer que los peces fueron transportados enteros, pues la suma de los huesos del esqueleto apendicular ${ }^{6} \mathrm{y}$ del cráneo, con 476 elementos anatómicos (47\%), tiene una correspondencia proporcional con los 533 huesos de la columna vertebral (53\%).

En lo que respecta a las 30 placas subdérmicas de cocodrilo, muy probablemente de Crocodylus moreletii ${ }^{7}$, se puede hacer mención a lo que López Austin (1996) propone como Cipactli y a su presencia como cocodrilo en la iconografía de Teotihuacan, la cual queda de manifiesto al observar las figuras zoomorfas que representan a «el que tiene púas o espinas», en la Pirámide de Quetzacoatl, mencionando que las

\footnotetext{
5 Nos referimos con «indirecto» al método empleado en la preservación de los peces, seguramente por el salado, que al utilizar sales marinas o minerales, incluían los elementos antes mencionados, sin que de ello estuvieran conscientes quienes aplicaran la técnica de preservación.

6 Resulta interesante mencionar que tanto las aletas como las agallas, son elementos que inmediatamente son desprendidos en una pescadería, cuando se requiere su consumo en fresco; situación que no ocurre cuando el pez es tratado, ahumado o salado, pues en mercados actuales se pueden observar completos, abiertos ventralmente, conservando los apéndices.

7 Fueron recuperadas en el área de actividad 96 (AA96), relleno 1 (R1), y relleno 2 sobre piso 2 (R2/P2) del cuarto 262B (C262B), correspondiente a una cala de saqueo moderna, por lo que sin duda se perdió una valiosa información respecto a la presencia de este animal en el contexto arqueológico teotihuacano.
} 
figuras de Tláloc son en realidad hombres con máscaras de cocodrilos o tocados del monstruo de tierra. Otro dato relevante es el que menciona Seler (2008: 231):

«El Cipactli de los mexicanos siempre está caracterizado por una hilera de púas o espinas sobre la espalda; con frecuencia también tiene estas púas sobre los miembros (figs. 659 y 672). Casi siempre están claramente marcadas las características del cocodrilo».

Bajo los argumentos anteriores la presencia de las placas subdérmicas de cocodrilo en el sitio de estudio, sin encontrar ningún otro elemento anatómico, puede tener su explicación a partir de que dichas placas formaran parte de una piel curtida para adornar atavíos o indumentarias, que seguramente se confeccionaban en el lugar (Manzanilla et al. 2009; Rodríguez 2006).

Otro organismo costeño que se identificó en Teopancazco es el erizo de mar (Eucidaris thouarsii); su presencia se establece a partir de la identificación de una espina tallada en el R4 del C158B, en el sector 10, siendo éste un elemento aislado que no puede dejarse de mencionar, ya que es el primero en su tipo para Teotihuacan; al respecto menciona el Dr. Solís (2005):

«Esta especie de erizo de mar se observa desde la costa occidental de Baja California, México, hasta Ecuador. Es una especie de erizo de aguas someras del pacífico tropical»

Es decir, se trata de un animal de aguas poco profundas, lo cual implicaría que su captura sería sencilla para quienes visitan las costas, o bien para quienes lo utilizan como fuente de pigmento natural; al respecto el Dr. Solís (2005) menciona:

«Una revisión bibliográfica del uso de los erizos me condujo a conocer que en las zonas costeras, las espinas de estos equinoideos son talladas para obtener de ellos un pigmento azul-purpúreo».

La reducción de la espina de erizo de mar es notable y ciertamente su presencia se puede deber a que ésta pudo haber sido tallada para obtener el pigmento; sin embargo, también se tiene la posibilidad de que la espina haya sido un dije u objeto decorativo, fijado de alguna manera a un atuendo o traje. El uso de espinas de erizo de mar como elemento utilitario en atavíos no es un hecho aislado, como ilustra un comentario realizado por el Mtro. Edmundo Teniente:

«Recuerdo este tipo de espina, trabajada de manera similar, en las vestimentas ceremoniales de una tribu del sur de Estados Unidos, los cuales son exhibidas en el Museo Smithsonian» (Rodríguez 2006: 195).

En lo que se refiere a las pinzas de cangrejos rojos (Gecarcinus ca. lateralis) y a la de cangrejo azul (Cardisoma ca. guanhumi), éstas fueron encontradas en diferentes cuartos, específicamente en las áreas de actividad 154 y 88 . Estos organismos tienen una amplia distribución en las costas del Golfo de México, Yucatán y el Caribe mexicano.

En el primero de ambos casos, Gecarcinus ca. lateralis, es un animal que suele ser consumido por el hombre y no se descarta la posibilidad de que sus pinzas, después de su consumo, hayan sido empleadas como objetos que adornaran algún tipo de vestimenta o simplemente como colgante; al respecto el Dr. Ramiro Román (2005) menciona: 
«El color de los especímenes es marrón, con la región dorsal del caparazón generalmente negra; las patas ambulatorias son marrón claro con las quelas (pinzas) rojizas».

Las características anteriores posiblemente fueron factor para que las personas se interesaran en este organismo y guardaran las pinzas con el propósito de poseer un dije que llamara la atención. Esta situación no debió de ser muy diferente para $C$. guanhumi, del cual el Dr. Román (2005) menciona:

«Esta especie se conoce comúnmente como cangrejo azul de tierra o cangrejo blanco, su color varía en el dorso y costados del cuerpo desde azul grisáceo hasta anaranjado y blanco, lo cual depende de la edad».

En lo que se refiere al uso alimentario de los cangrejos, existen investigaciones que evidencian su aprovechamiento para tales fines; sin embargo éstas son, por lo general, en sitios costeros; por ejemplo en la costa de Huaca Prieta en Perú se detectó, en un análisis de coprolitos humanos, restos de cangrejo, indicando que su uso tiene más que ver con el alimento; a propósito de lo anterior Callen (1980: 241) menciona que «El cangrejo y el erizo de mar, así como las frutas de temporada, probablemente tenían más bien el carácter de manjares que de alimentos básicos».

Con base en lo anterior cabe la posibilidad de que los cangrejos fueran usados primariamente como manjar en algún evento especial y, posteriormente, el exoesqueleto, o parte de él, como las pinzas, pudiera haber sido empleado para adornar atuendos o como simple colgante, pues la captura, en ambas especies, es tan sencilla que basta con ir a un lugar en donde la población sea abundante para colectarlos con las manos, tal y como se puede hacer hoy en día, aprovechando sus hábitos semiterrestres.

\section{Procedencia de los recursos costeros}

Prácticamente todas las especies identificadas habitan la zona costera del Golfo de México, situación que resuelve el importante aspecto de hacia dónde estaba dirigido el esfuerzo que involucraba la captura y manejo de todos estos animales y/o sus partes; con base en lo anterior ¿es posible inferir el lugar de origen de los recursos costeros encontrados en Teopancazco? A lo largo de la costa del Golfo de México se pueden observar vestigios de culturas como la olmeca, maya y teotihuacana, así que es evidente que los peces identificados en Teopancazco, proceden de algún lugar de esta costa, dado que es sabido que el estado teotihuacano ejerció su control en esta región de Mesoamérica, dejando huella de su paso por diferentes regiones. Así, el intercambio de bienes costeros debió ser factor importante en la vida económica de Teotihuacan, ya sea porque estaban directamente bajo su control, o porque sus nexos comerciales la obligaban a tener enclaves en otros lugares. Ciertamente, durante el horizonte Clásico, Teotihuacan fue determinante en el desarrollo de muchas comunidades contemporáneas (Matos 2000). Así se puede mencionar que Teotihuacan mantenía relaciones con zonas del Altiplano Central, como Otumba o la Sierra de las Navajas -donde se obtenía obsidiana verde-, siguiendo una ruta bien definida que atravesaba sitios como Tepeapulco y Huapalcalco, en el estado de Hidalgo, siguiendo de ahí hacia la Costa del Golfo, en donde existían gran cantidad de recursos de origen 
tropical que buscaba controlar; aunado a lo anterior también se ha establecido que la cultura teotihuacana centraba su interés en los yacimientos de calizas de lugares como Chingú y el Sitio 83, cerca de Tula, donde existen asentamientos teotihuacanos que probablemente jugaban un papel relevante en el control del área (Matos 2000).

La relación entre Teotihuacan y la Costa del Golfo fue reconocida desde principios del siglo XX cuando Seler, Mena, Krickerberg y Beyer notaron la similitud entre ambas regiones por la presencia de volutas entrelazadas y por los atributos encontrados en las figurillas y, posteriormente, cuando Du Solier y Valenzuela hallaron evidencia de imitaciones de motivos estilísticos teotihuacanos en la cerámica de fabricación local. A partir de entonces, diversos elementos han sido encontrados en sitios como Chachalacas, Xiutetelco, Altotonga, El Pital, Napatecuhtlan, Nopiloa, Cerro de las Mesas, Tres Zapotes, Matacapan, La Joya, entre otros (Mejía 2008: 64). Entre estos asentamientos destaca uno que pudo haber servido de enclave teotihuacano, Matacapan, sitio que ocupa una posición importante, ya que pudo ser paso obligado hacia la costa. Entre los rasgos teotihuacanos aquí presentes se tiene arquitectura talud-tablero, áreas residenciales con cuartos y corredores alrededor de patios, la orientación de los muros tiene diferencia de un grado en comparación con los de Teotihuacan, entierros flexionados debajo de los pisos y cerámicas muy semejantes (Matos 2000).

Con base en lo anterior se presume que Teotihuacan pudo haber tenido interés en enviar a un grupo de emisarios (¿comerciantes?) a instalarse en esa región, por razones económicas, pues este sitio se encuentra cerca de yacimientos de piedra volcánica de alta calidad y de arcillas para hacer cerámica de pasta fina, además de que en ellos se tenían terrenos de cultivo de algodón. Pudo asimismo servir de paso hacia la zona maya, principalmente para quienes se dirigían hacia la península de Yucatán o a las Tierras Bajas peteneras como las de Tikal (Mejía 2008; Rodríguez 2010).

Existen otros sitios arqueológicos de la costa veracruzana donde están presentes evidencias de influencia teotihuacana; así en el Cerro de las Mesas se han localizado copias de formas cerámicas teotihuacanas, candeleros, copas, floreros y cerámica Anaranjada Delgada.

Lo anterior aumenta la probabilidad de que el recurso costero haya procedido de las costas veracruzanas, sobre todo de la porción norte, pues la alta concentración de pez bobo (Joturus pichardi), el diente de un tiburón toro (Carcharinus leucas), ambos cangrejos (Cardisoma ca. guanhumi y Gecarcinus ca. lateralis) y cocodrilo (muy posiblemente Crocodylus moreletii), así lo hacen patente.

Sobre este mismo aspecto, Daneels (2002) propone dos tipos de contacto entre Teotihuacan y La Joya, Veracruz: el primero tiene lugar entre los años 100 y 300 d.C., cuando Teotihuacan toma elementos de contextos de prestigio del centro-sur de Veracruz; el segundo se da entre el 300 y 600 d.C. cuando la relación cambia y se pierde el carácter de prestigio a favor del comercial. Partiendo de lo anterior, la propuesta, apoyada en las investigaciones de Rattray (1998) y Morante (2004), es que los recursos pesqueros seguían dos rutas hacia Teotihuacan y/o la Cuenca de México:

\footnotetext{
1. Teotihuacan $\rightarrow$ Zacatepec $\rightarrow$ Perote $\rightarrow$ Serafín $\rightarrow$ Cuajilote $\rightarrow$ El Pital $\rightarrow$ Las Higueras $\rightarrow$ Golfo de México.

2. Teotihuacan $\rightarrow$ Maltrata $\rightarrow$ Nopiloa $\rightarrow$ Dicha Tuerta $\rightarrow$ Tlacotalpan $\rightarrow$ Matacapan $\rightarrow$ Área maya.
} 


\section{Captura y preparado de los peces}

Como se ha podido observar, la concentración de peces bobo es mayor a la del resto de los otros organismos, es decir, se tienen evidencias suficientes para proponer que:

1. En una primera instancia, la captura se efectúa con el propósito de atrapar a este tipo de pez, aunque es muy probable que al momento de efectuar la pesca se hayan capturado en la red algunas otras variedades que no estaban consideradas.

2. En una segunda instancia se puede argumentar que la pesca se efectuó en alguna temporada en la cual el pez bobo era el más abundante.

En el primero de ambos casos, los pescadores tienen por encomienda capturar una determinada variedad de pez (en este caso pez bobo); sin embargo al estar en las labores de pesca se capturó también una cierta cantidad de huachinangos y otras variedades, y aunque no eran el objetivo principal, tampoco se desdeñó su «captura accidental». En el segundo caso la idea está más encaminada a que el momento de la actividad pesquera, por el ciclo de vida del pez bobo como organismo catádromo ${ }^{8}$, y su abundancia respecto a las otras variedades de peces, puede establecerse entre los meses de enero a marzo, ya que es en el primer mes del año cuando las hembras comienzan a cargarse de huevecillos.

Caso contrario sucede con la barracuda y el tiburón, los cuales por ser animales que imponen ante su sola presencia, pareciera que éstos más bien fueron capturados para alguna actividad ritual $u$ ornamental, como sucedería con las barracudas en el primero de los casos, o para obtener de ellos elementos, como el diente de tiburón, que pudieran servir de adorno; cualquiera que sea el motivo resulta interesante la cita que hace Brockmann respecto a las creencias que se tienen sobre los peces:

«Entre las creencias religiosas relacionadas con la pesca aparece el numen del dueño de los peces, que es una forma especial del dueño de los animales» (Brockmann 2004: 150).

Así entre los totonacas, por ejemplo, los sacrificios se ofrecen al «dueño del agua» para tener suerte en la pesca; mientras que entre los popolucas de Veracruz el dueño de los peces lleva el nombre de «chanco»y a él es a quien están destinadas las actividades rituales.

Con base en lo anterior, y salvo la captura del tiburón y la barracuda, que seguramente se efectuó con el uso de arpón y como se haría hoy en día con un grupo de personas, las fuentes del siglo XVI señalan el empleo constante de redes, en diferentes modalidades:

«Los instrumentos más comunes de que se servían los mexicanos para la pesca, eran las redes, pero usaban también de anzuelos, arpones y mazas» (Clavijero 1991: 235).

Aun así también hay que señalar el antecedente del capitán Francisco Hernández de Córdoba, citado por Villanueva (Informe Cha Kan Putun) quien menciona, al ins-

\footnotetext{
8 Los organismos catádromos son aquellos que nacen en el mar, después migran a los ríos, donde se desarrollan y vuelven al mar a desovar cuando alcanzan la madurez sexual.
} 
peccionar la costa de la península, una aldea con «cerca de ocho mil casas y dos mil canoas»; es decir la probabilidad de que se emplearan redes y como transporte canoas es, desde nuestro muy particular punto de vista, muy alta.

Respecto a la preparación, los resultados indican que los peces llegaron completos, es decir, no fueron fileteados ni seccionados, pero sí eviscerados con el propósito de evitar la descomposición ${ }^{9}$ y la rigidez cadavérica o rigor mortis, misma que puede producirse en un lapso de entre una y diez horas.

Partiendo del hecho anterior, se considera poco admisible que los peces se transportaran frescos, más aun si consideramos la cantidad de NMI obtenidos (99), que en su mayoría son ejemplares de peces bobo y huachinangos, organismos que, por la comparación de los huesos con ejemplares actuales, sobrepasan los $45 \mathrm{~cm}$ de longitud total. La hipótesis es que estos animales fueron preparados, salados o ahumados, para su traslado a Teopancazco, con métodos de preservación similares a los que podemos observar actualmente en diversos mercados tradicionales del centro y costa del Golfo de México, donde se ofrecen preparados en hojas de maíz, como si fuesen tamales, después de haber sido ahumados, cocidos sobre las brasas, salados y secados al sol.

\section{Ecosistemas de procedencia de la fauna costera}

Los peces identificados en el sitio de estudio tienen una alta probabilidad de provenir de lagunas costeras, situación que resulta importante señalar, pues se trata de un ecosistema muy heterogéneo que permite el intercambio dinámico de energía entre la tierra costera, sus ríos de agua dulce, el mar y la atmósfera circundante, favoreciendo las interacciones físicas y biológicas que promueven alta diversidad biológica que incluye, entre otros, crustáceos, moluscos, aves, y por supuesto peces en diferentes etapas de sus ciclo de vida (Rodríguez 2006: 198).

No obstante el gran avance que representa la delimitación de la región de origen de los recursos costeros descubiertos en Teopancazco a una porción de Veracruz y de cómo estos animales eran preparados para su largo viaje hacia Teotihuacan, es factible delimitarlo más en función de las características ecológicas de las especies descubiertas y las tradiciones actuales sobre su uso.

De acuerdo con la biología de las especies de peces descubiertas, todas ellas tienen un ciclo de vida dentro del cual las zonas costeras juegan un papel relevante, pues en las fases tempranas ocupan preferente o exclusivamente estos ambientes. El pez bobo es de hábitos dulceacuícolas, ocupando las partes bajas de los ríos, estuarios y lagunas en la época seca, mientras que el resto es de hábitat marino y se encuentran en estos mismos espacios en temporada reproductiva. Considerando las características biogeográficas de la zona, sin duda las lagunas costeras fueron el ambiente ideal para la pesca por parte de los habitantes de la región, pues en ellas tenían acceso a estos recursos ícticos con poco esfuerzo.

\footnotetext{
9 Hay que tener presente que el evisceramiento tiene como propósito, como hoy en día, detener todos los procesos bioquímicos de la putrefacción.
} 
Respecto de los cangrejos, el Cardisoma ca. guanhumi habita todo el año en manglares, lagunas y pantanos, abandonando estos espacios en la temporada de lluvias, es decir, de junio a septiembre, para ir a desovar a las playas. Respecto al segundo, especie Gecarcinus ca. lateralis, su esquema de vida es similar, pero es entre abril y junio cuando avanza hacia las playas con el mismo propósito. De esta forma, y aunque las temporadas reproductivas de las dos especies varían, ciertamente es al inicio de la temporada de lluvias, en decir, en junio, cuando ambos cangrejos se hacen visibles para el hombre, durante sus jornadas migratorias hacia el mar.

En el caso del cocodrilo de pantano, Crocodylus moreletii, su distribución abarca desde el estado de Tamaulipas hasta Guatemala. Su abundancia se ha visto afectada en la actualidad, sin embargo su hábitat siempre ha estado sujeto a los cuerpos de agua como son los pantanos, riachuelos, lagunas costeras, ciénagas o marismas, donde con seguridad se le podía observar durante todo el año. En la mayoría de las ocasiones las hembras alcanzan la madurez sexual a los 10 años de edad, tiempo en el cual se inicia la reproducción, una vez al año, iniciando en febrero y terminando en mayo.

Partiendo de lo anterior, y tomando de base la lista de peces reconocidos como altamente comestibles como los huachinangos (Lutjanus sp), el robalo (Centropomus sp) o la mojarra plateada (Eucinostomus sp), que son especies muy apreciadas dentro de la cocina mexicana en la actualidad, los resultados demuestran que el empleo de estas especies pesqueras en la dieta del habitante de estas tierras tiene una historia milenaria, uso alimentario que comparten con las dos especies de cangrejos.

Así, cuando se habla acerca de la explotación de los recursos marinos en tiempos prehispánicos, generalmente llegamos a un espacio en donde hay muy poca información, sin importar si se trata del Golfo de México, el Caribe o la costa del Pacífico. Sin embargo la idea general es que la tradición pesquera fue escasa y estrictamente limitada a quienes habitaron las costas y por tanto se ha concedido poca importancia a la búsqueda de datos respecto a la manera en que eran aprovechados los recursos marinos. Sabemos que muchos de los moluscos eran obtenidos en las playas o pocos metros mar adentro, pero no es lo mismo colectar caracoles que perseguir a un pez en su ambiente, lo cual nos obliga a ubicar cuál era el esquema que les permitía acceso al recurso.

Los datos actuales y etnohistóricos (Rodríguez 2010) proporcionan abundante información acerca de los medios por los cuales se capturaban los peces, desde redes hasta arpones, empero poco se dice respecto de si se trataba de pesca en mar abierto o no. La información derivada de este estudio permite concluir que el hábitat explotado por las comunidades humanas para aprovechar el recurso era fundamentalmente la laguna costera, la cual es muy común en esa región de la costa de Veracruz y, como se indicó, es un espacio ecológico en el cual se concentran las especies registradas, incluidos los cangrejos y el cocodrilo. En función de esto es posible afirmar que, desde que el hombre llegó a esta zona, vio el potencial de estas lagunas y al paso de los siglos se constituyó un conjunto de peces cuyo empleo como alimento alcanzó suficiente valor para adquirir identidad cultural, y así pasar a ser parte del acervo de la tradición culinaria del mexicano, suerte que no corrió en pez bobo (Joturus pichardii), pues su consumo es, hoy en día, muy local en la costa central veracruzana. 


\section{Cosmovisión costera en la Ciudad de los Dioses}

Sin duda el dato de que la mayoría de las especies de peces identificadas constituyen en la actualidad parte de la tradición alimentaria de México es una fuerte evidencia de que su presencia en Teopancazco se relaciona con su consumo, aspecto que se refuerza por las sales reconocidas en algunos huesos; sin embargo su sola condición de fuente de alimento no justifica el esfuerzo que necesariamente debió hacerse para llevarlos hasta Teotihuacan. Poco importa si se trataba de ejemplares preparados o no, de individuos completos o sus partes o qué tan frecuente era su flujo hacia la ciudad; simplemente debemos afirmar que el aspecto alimentario no es suficiente para entender la presencia de estos animales.

Considerando que la mayoría de las especies reconocidas tienen ciclos de vida que incluyen su presencia en las lagunas costeras en épocas definidas del año, sin duda su llegada y nivel de abundancia en tal o cual momento era visto como un medio de comunicación entre el hombre y los dioses. En todos los meses del año existe alguna de las especies en estos ambientes (Cuadro 3), lo cual les daría un valor específico, pero también es interesante constatar que la mayor concentración se da entre junio y agosto, es decir en la primera parte de la temporada de lluvias, el cual es también el periodo en el que el maíz se encuentra en desarrollo y por tanto un momento crucial dentro del ciclo agrícola.

Desde esta perspectiva la presencia de estos animales en Teopancazco y su empleo, fuera de la forma que fuera, debía tener un valor religioso ligado con la agricultura.

Curiosamente el pez bobo, el más abundante en la colección de Teopancazco, ocupa lagunas costeras en la época de secas, condición que le daría el papel de contraparte de los demás y ser considerado como parte del ciclo agrícola, pero para el periodo que incluye desde la cosecha hasta el inicio del barbecho.

Pero hay más aún, en este sitio fueron descubiertas varias pinturas murales que representaban actos de procesión de sacerdotes relacionados con el ciclo agrícola (Figura 3). En ellas se presentan a estos personajes con vestimentas muy decoradas y

Cuadro 3: Taxa de peces y cangrejos con ciclos reproductivos estacionales y época del año en la cual están presentes en las lagunas costeras.

\begin{tabular}{|c|c|c|c|c|c|c|c|c|c|c|c|c|}
\hline \multirow[t]{2}{*}{ Taxa } & \multicolumn{12}{|c|}{ Meses } \\
\hline & $\mathbf{E}$ & $\mathbf{F}$ & $\mathbf{M}$ & $\bar{A}$ & $\mathbf{M}$ & $\mathbf{J}$ & $\mathbf{J}$ & $\mathbf{A}$ & $\mathbf{S}$ & $\mathbf{O}$ & $\overline{\mathbf{N}}$ & D \\
\hline Joturus pichardi & - & $\bullet$ & $\bullet$ & $\bullet$ & & & & & & & & $\bullet$ \\
\hline Centropomus & & & & & $\bullet$ & - & $\bullet$ & $\bullet$ & $\bullet$ & & & \\
\hline Mycteroperca bonaci & & & & & & & $\bullet$ & $\bullet$ & & & & \\
\hline Lutjanus & & & & & & - & - & $\bullet$ & - & $\bullet$ & $\bullet$ & \\
\hline Dipterus & & & & $\bullet$ & $\bullet$ & - & - & $\bullet$ & & & & \\
\hline Bairdiella ronchus & $\bullet$ & & & & & & & & & & & • \\
\hline Sphyraena barracuda & & & & & & - & - & $\bullet$ & $\bullet$ & & & \\
\hline Cardisoma guanhumi & & & & & & $\bullet$ & $\bullet$ & $\bullet$ & $\bullet$ & & & \\
\hline Gecarcinus lateralis & & & & $\bullet$ & $\bullet$ & $\bullet$ & & & & & & \\
\hline TOTAL & 2 & 1 & 1 & 3 & 3 & 6 & 6 & 6 & 4 & 1 & 1 & 2 \\
\hline
\end{tabular}




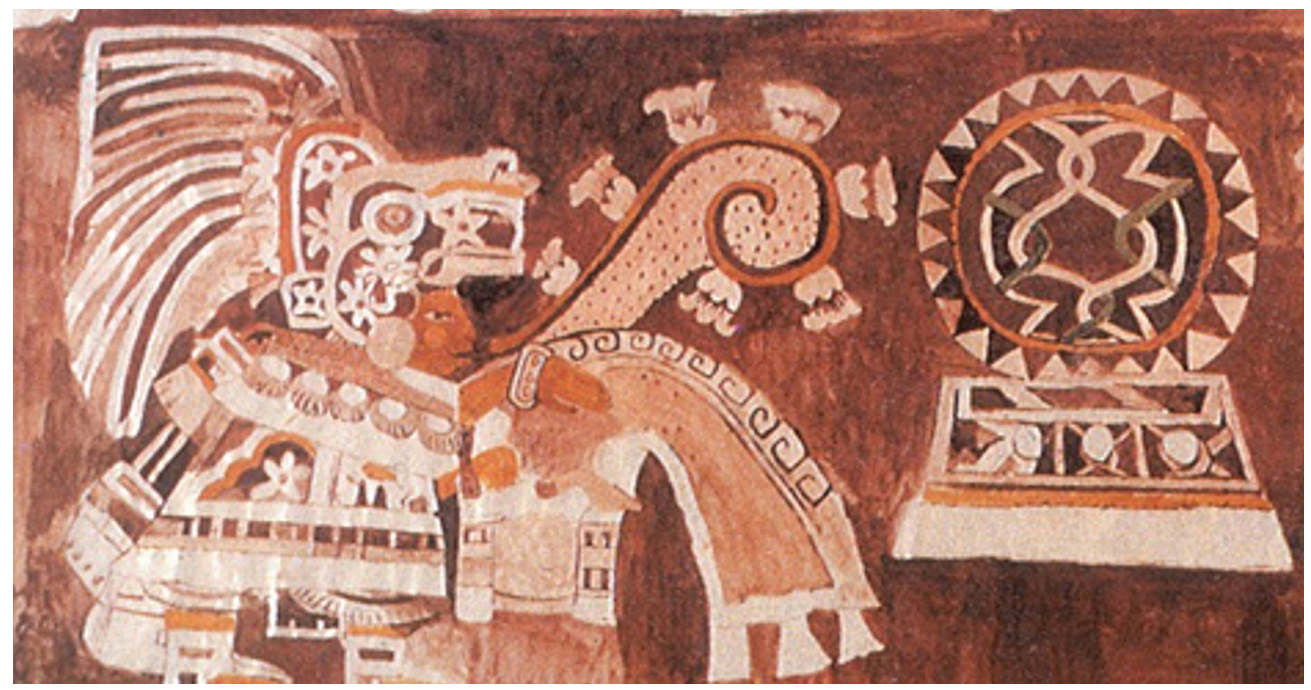

Figura 3: Pintura mural del sitio de Teopancazco en la cual se muestra a un sacerdote en un acto de procesión, junto a un altar (derecha) con una vestimenta que incluye elementos alusivos al mar tales como estrellas y conchas (Manzanilla et al. 2009); el acto incluye oraciones (vírgula que sale de la boca) mientras arroja semillas con la mano derecha, indicando así la relación entre los contextos agrícola y marino.

parte del mismo incluye elementos como ondas de agua, estrellas, muy probablemente de mar, y conchas marinas (Manzanilla et al. 2009).

Si unimos esta información a la anterior podemos reconocer la relación entre lo marino, lo agrícola y lo ritual, dentro de lo cual es factible proponer, sin mayor problema, que parte de estos ritos incluyeran el consumo de estos peces, por supuesto, dentro de un marco simbólico.

\section{Consideraciones finales}

La arqueozoología mexicana es un área de investigación en la cual el enfoque interdisciplinario es no sólo deseable, sino indispensable, ya que entender cómo era aprovechado un recurso animal implica verlo desde múltiples puntos de vista, desde lo estrictamente biológico y terrenal hasta lo más simbólico y abstracto. Reconocer el uso de un recurso no tiene gran valor si sólo nos limitamos a la afirmación de que se utilizaba; antes bien, es indispensable compenetrarnos en las bases culturales sobre las cuales se asentaba la valoración del mismo para comprender el impacto que tenía dentro de la cultura estudiada. Paradójicamente, conforme avanzamos en este juego en el cual lo biológico y lo antropológico se van alternando dentro de nuestra búsqueda de información, más necesitamos entender los procesos naturales para explicar las pautas culturales, en un esquema que finalmente sea acorde con la realidad de que realizamos trabajo arqueozoológico en una región donde la riqueza biológica y cultural se ubican entre lo más destacable del planeta. 
Agradecimientos: Queremos agradecer a la doctora Linda Manzanilla por las facilidades otorgadas para trabajar con los materiales arqueozoológicos de su proyecto: Teotihuacan: elite y gobierno. Por las asesorías y comentarios a los Dres. Ramiro Román, Francisco Solís y Ana Fabiola Guzmán, así como a los Mtros. Mario Martínez, Edmundo Teniente y Alicia Blanco.

\section{Referencias bibliográficas}

BÖKÖNYI, Sandor

1970 «A New Method for the Determination of the Number of Individuals in Animal Bone Material». American Journal of Archaeology 74: 291-292.

Brokmann, Andreas

2004 La pesca indígena en México. México: Instituto de Investigaciones Antropológicas, Universidad Nacional Autónoma de México.

Callen, Erick O.

1980 «La dieta revelada por los coprolitos», en Ciencia en antropología, Don Brothwell y Eric Higgs, comps., pp. 240-248. México: Fondo de Cultura Económica.

Clavijero, Francisco Javier

1991 Historia antigua de México. México: Editorial Porrúa.

DANEELS, Annick

2002 «Presencia de Teotihuacan en el sur y centro de Veracruz», en Ideología y política a través de materiales, imágenes y símbolos. Memoria de la Primera Mesa Redonda de Teotihuacan, $\mathrm{M}^{\mathrm{a}}$ Elena Ruiz Gallut, ed., pp. 655-683. México: Instituto de Investigaciones Antropológicas (UNAM) - Instituto de Investigaciones Estéticas (UNAM) - Instituto Nacional de Antropología e Historia.

Gamio, Manuel

1922 La población del valle de Teotihuacan. El medio en que se ha desarrollado su evolución étnica y social. Iniciativas para procurar su mejoramiento. México: Dirección de Antropología, Secretaria de Agricultura y Fomento - SEP.

Lombardo DE Ruíz, Sonia

1996 «El estilo teotihuacano en la pintura mural», en La pintura mural prehispánica en México. Teotihuacan, Vol. II, Tomo II, Estudios, Beatriz de la Fuente, coord., pp. 3-64. México: Instituto de Investigaciones Estéticas, Universidad Nacional Autónoma de México.

López Austin, Alfredo

1996 «La cosmovisión mesoamericana», en Temas mesoamericanos, Sonia Lombardo y Enrique Nalda, coords., pp. 471-500. México: Instituto Nacional de Antropología e Historia - CONACULTA.

Manzanilla Naim, Linda R.

1997 Informe Técnico del Proyecto «Teotihuacan: elite y gobierno, excavaciones en Teopancazco». Entregado al Consejo de Arqueología.

2006 «Estados corporativos arcaicos. Organizaciones de excepción en escenarios excluyentes». Cuicuilco 13 (36): 13-45.

2007 «Las 'casas' nobles de los barrios de Teotihuacan: Estructuras exclusionistas en un 
entorno corporativo», en Memorias de la XXVIII Mesa Redonda de la Sociedad Mexicana de Antropología, pp. 453-470. México: El Colegio Nacional.

Manzanilla Naim, Linda R., Raúl Valadez, Bernardo Rodríguez, Gilberto Pérez, Adrián VelÁZQUEZ y Norma VALENTín

2009 «Producción de adornos y atavíos del poder en Teotihuacan», en Memorias del Simposio ICA 2009: Producción de Bienes de Prestigio Ornamentales y Votivos de la América Antigua, Emiliano Melgar coord. México: Universidad Iberoamericana. (Versión en CD).

Matos Moctezuma, Eduardo

2000 El milenio teotihuacano. Pasajes de La Historia, 4. México: México Desconocido.

MeJia ApPEL, Gabriela Inés

2008 Análisis de paleodieta en Teopancazco. Un acercamiento a las diferencias alimenticias del clásico teotihuacano. Tesis de Licenciatura en Arqueología. México: Escuela Nacional de Antropología e Historia.

MiLlon, René

1973 Urbanization at Teotihuacan, México. Volume One: the Teotihuacán Map. Austin: University of Texas Press.

Morante, Rubén

2004 «Del Altiplano a la Costa del Golfo: intercambios culturales y materiales», en $L a$ Costa del Golfo en tiempos teotihuacanos: problemas y perspectivas. Memorias de la Segunda Mesa Redonda de Teotihuacan, Mª Elena Ruíz Gallut y Arturo Pascual Soto eds., pp. 23-43. México: Instituto Nacional de Antropología e Historia.

O'CONNOR, Terry

2000 The Archaeology of Animal Bones. Stroud: Sutton Publishing Limited.

PADRÓ IRIZARRY, Johana

2002 La industria del hueso trabajado en Teotihuacan. Tesis de Doctorado en Antropología. México: Facultad de Filosofía y Letras, Instituto de Investigaciones Antropológicas, Universidad Nacional Autónoma de México.

PECCI, Alessandra

2000 Análisis químico de pisos y áreas de actividad. Estudio de caso en Teopancazco, Teotihuacan. Tesis de Maestría en Antropología. México: Facultad de Filosofía y Letras, Instituto de Investigaciones Antropológicas, Universidad Nacional Autónoma de México.

Polaco, Óscar y Ana Fabiola Guzmán

1997 Arqueoictiofauna mexicana. México: Instituto Nacional de Antropología e Historia.

RATTRAY, Evelyn

1998 «Rutas de Intercambio en el periodo Clásico en Mesoamérica», en Rutas de Intercambio en Mesoamérica. III Coloquio Pedro Bosch-Gimpera, Evelyn Rattray ed., pp. 77-100. México: Instituto de Investigaciones Antropológicas, Universidad Nacional Autónoma de México.

Rodríguez Galicia, Bernardo

2006 El uso diferencial del recurso fáunico en Teopancazco, Teotihuacan, y su importancia en las áreas de actividad. Tesis de Maestría en Antropología. México: Fa- 
cultad de Filosofía y Letras, Instituto de Investigaciones Antropológicas, Universidad Nacional Autónoma de México.

2010 Captura, preparación y uso diferencial de la ictiofauna encontrada en el sitio arqueológico de Teopancazco, Teotihuacan. Tesis de Doctorado en Antropología. México: Facultad de Filosofía y Letras, Instituto de Investigaciones Antropológicas, Universidad Nacional Autónoma de México.

RomÁn, Ramiro y Mario MARTínez

2005 Reporte de identificación de fragmentos de crustáceos realizada por personal de carcinoparasitología, del Instituto de ciencias del mar y Limnología, para el Instituto de Investigaciones Antropológicas-UNAM. Entregado a la Dra. Linda Manzanilla, dentro del seminario permanente de Teopancazco Instituto de Investigaciones Antropológicas (UNAM); Instituto de Ciencias del Mar y Limnología (UNAM).

Rzedowski Roter, Jerzy y Graciela CALDERÓn de RzEDOwski

1979 Flora fanerogámica del Valle de México, Vol.1. México: C.E.C.S.A.

SELER, Eduard

2008 Las imágenes de los animales en los manuscritos mexicanos y mayas. México: Casa Juan Pablos.

Solís, Francisco

2005 Nota sobre la identificación de erizo de mar (Eucidaris thouarsii). Entregado a la Dra. Linda Manzanilla, dentro del seminario permanente de Teopancazco Instituto de Investigaciones Antropológicas (UNAM); Instituto de Ciencias del Mar y Limnología (UNAM).

VALADEZ AzÚA, Raúl

1983 Paleoecología de la cuenca de México durante el Pleistoceno superior. Tesis de Licenciatura. México: Facultad de Ciencias, Universidad Nacional Autónoma de México.

1992a Impacto del recurso faunístico en la sociedad teotihuacana. Tesis de Doctorado en Ciencias Biológicas. México: Facultad de Ciencias, Universidad Nacional Autónoma de México.

1992b «Vertebrados alóctonos encontrados en la antigua ciudad de Teotihuacan». Ciencia y Desarrollo 18 (106): 49-61.

1993 «Macrofósiles faunísticos», en Anatomía de un conjunto residencial teotihuacano en Oztoyohualco, Linda Manzanilla coord., Vol. II, pp. 729-813. México: Instituto de Investigaciones Antropológicas, Universidad Nacional Autónoma de México.

2011 «Una ciudad prehispánica vista a través de la fauna», en Memorias del Congreso Internacional «Culturas Americanas y su Ambiente: Perspectivas desde la Zooarqueología, Paleobotánica y Etnobiología», Christopher Götz coord. México: Universidad Autónoma de Yucatán. (En prensa).

Villanueva García, Gerardo

2000 Cha Kan Putun. Un puerto prehispánico en el Golfo de México. Análisis del material malacológico. Sección de Biología. Informe Técnico. México. 